\title{
FREQUENTLY HYPERCYCLIC SEMIGROUPS
}

\author{
ELISABETTA M. MANGINO ${ }^{1}$ AND ALFREDO PERIS ${ }^{2}$
}

\begin{abstract}
We study frequent hypercyclicity in the context of strongly continuous semigroups of operators. More precisely, we give a criterion (sufficient condition) for a semigroup to be frequently hypercyclic, whose formulation depends on the Pettis integral. This criterion can be verified in certain cases in terms of the infinitesimal generator of semigroup. Applications are given for semigroups generated by Ornstein-Uhlenbeck operators, and especially for translation semigroups on weighted spaces of $p$-integrable functions, or continuous functions that, multiplied by the weight, vanish at infinity.
\end{abstract}

MSC2010: 47A16, 47D06

Keywords: Chaotic $C_{0}$-semigroups, frequently hypercyclic $C_{0}$-semigroups, translation semigroups.

\section{INTRODUCTION}

The hypercyclic behaviour of strongly continuous one parameter semigroups was studied in a systematic way for the first time in the paper by Desch, Schappacher, and Webb [21. They gave a sufficient condition for hypercyclicity of a semigroup based on the analysis of the point spectrum of the generator of the semigroup. Moreover they characterized hypercyclic translation semigroups defined on weighted spaces of continuous or integrable functions on the real line. However, during the last years it was shown that hypercyclicity appears in $C_{0}$-semigroups associated to "birth and death" equations for cell populations, transport equations, first order partial differential equations and diffusion operators as Ornstein-Uhlenbeck operators (see [13] for a survey on the subject. Further references on hypercyclic semigroups and related properties are, e.g., 2, 3, 7, 8, 9, 16, 18, 20, 24, 28, 29, 30, 31, 32, 34, 36, 37]).

We recall that, if $X$ is a separable infinite-dimensional Banach space, a $C_{0}$-semigroup $\left(T_{t}\right)_{t>0}$ of linear and continuous operators on $X$ is said to be hypercyclic if there exists $x \in X$ (called hypercyclic vector for the semigroup) such that the set $\left\{T_{t} x: t \geq 0\right\}$ is dense in $X$. An element $x \in X$ is said to be a periodic point for the semigroup if there exist $t>0$ such that $T_{t} x=x$. A semigroup $\left(T_{t}\right)_{t \geq 0}$ is called chaotic if it is hypercyclic and the set of periodic points is dense in $X$.

In [15], the second author, in collaboration with A. Conejero and V. Müller, proved that if $x \in X$ is a hypercyclic vector for $\left(T_{t}\right)_{t \geq 0}$ then for every $t>0$ the set $\left\{T_{n t} x\right.$ : $n \in \mathbb{N}\}$ is dense in $X$, i.e. $x$ is a hypercyclic vector for each single operator $T_{t}, t>0$. In particular, hypercyclicity is inherited by discrete subsemigroups. However, this is not the case in general if we change the index set [17], or if we consider the chaos property [5].

Motivated by Birkhoff's ergodic Theorem, Bayart and Grivaux introduced the notion of frequent hypercyclic operators [4] (see [6] and the references quoted therein, see also [10, 11, 27]), trying to quantify the frequency with which an orbit meets the 
open sets. This concept was extended to $C_{0}$-semigroups in [1]. We recall that the lower density of a measurable set $M \subset \mathbb{R}_{+}$is defined by

$$
\underline{\operatorname{Dens}}(M):=\liminf _{N \rightarrow \infty} \mu(M \cap[0, N]) / N,
$$

where $\mu$ is the Lebesgue measure on $\mathbb{R}_{+}$. A $C_{0}$-semigroup $\left(T_{t}\right)_{t \geq 0}$ is said to be frequently hypercyclic if there exists $x \in X$ such that Dens $\left(\left\{t \in \mathbb{R}_{+}: T_{t} x \in U\right\}\right)>0$ for any non-empty open set $U \subset X$.

If the lower density of a set $A \subset \mathbb{N}$ is defined by

$$
\underline{\operatorname{dens}}(A):=\liminf _{N \rightarrow \infty} \#\{n \leq N: n \in A\} / N
$$

an operator $T \in L(X)$ is said to be frequently hypercyclic if there exists $x \in X$ (called frequently hypercyclic vector) such that, for every non-empty open subset $U \subset X$, the set $\left\{n \in \mathbb{N}: T^{n} x \in U\right\}$ has positive lower density. In [15] it was proved that if $x \in X$ is a frequently hypercyclic vector for $\left(T_{t}\right)_{t \geq 0}$, then for every $t>0$ the $x$ is a frequently hypercyclic vector for the operator $T_{t}$.

In [10, 11], Bonilla and Grosse-Erdmann improve a result of Bayart and Grivaux and show the following Frequent Hypercyclicity Criterion for operators (See also 25] for a probabilistic criterion).

Proposition 1.1. Let $T$ be a continuous operator on a separable Banach space $X$. If there exist $X_{0} \subseteq X$, dense subset, and a map $S: X_{0} \rightarrow X_{0}$ satisfying:

(i) $T S x=x$, for all $x \in X_{0}$;

(ii) $\sum_{n=1}^{\infty} T^{n} x$ is unconditionally convergent for all $x \in X_{0}$;

(iii) $\sum_{n=1}^{\infty} S^{n} x$ is unconditionally convergent for all $x \in X_{0}$.

then $T$ is frequently hypercyclic.

The aim of the present paper is to give a continuous version of the frequent hypercyclicity criterion. The unconditional convergence of the series in Proposition 1.1 will be replaced by the Pettis integrability of orbits under the semigroup. Thanks to this criterion we will show that, e.g., the well-known Desch-Schappacher criterion for chaotic semigroups (see [21]) is actually a condition for frequent hypercyclicity. Moreover we prove that chaotic translation semigroups on weighted spaces of integrable functions defined on $[0,+\infty[$ are frequently hypercyclic. We give a necessary condition on the weight for frequently hypercyclicity. Since several properties of the Pettis integral are used in the proofs, for the convenience of the reader we recall in an appendix the main definitions and basic results.

\section{Frequent Hypercyclicity CRiterion for SEmigroups}

Proposition 2.1. Let $\left(T_{t}\right)_{t \geq 0}$ be a $C_{0}$-semigroup on a separable Banach space $X$. Then the following conditions are equivalent:

(i) $\left(T_{t}\right)_{t \geq 0}$ is frequently hypercyclic,

(ii) for every $t>0$ the operator $T_{t}$ is frequently hypercyclic.

(iii) there exists $t>0$ such that the operator $T_{t}$ is frequently hypercyclic.

Proof. The implication (i) $\Rightarrow$ (ii) was proved in [15]. It remains to prove that (iii) implies (i). Assume w.l.o.g. that $t=1$ and let $x$ be a frequently hypercyclic vector for $T_{1}$. Let $y \in X, U, V$ 0-neighbourhoods such that $V+V \subseteq U$. By the strong continuity of $\left(T_{t}\right)_{t \geq 0}$, there exists $0<\delta<1$ such that $T_{s} y-y \in V$ for every $s \in[0, \delta]$. 
Moreover, by the local equicontinuity of $\left(T_{t}\right)_{t \geq 0}$, there exists a 0-neighbourhood $V^{\prime}$ such that $T_{s}\left(V^{\prime}\right) \subseteq V$ for every $s \in[0, \delta]$.

By assumption,

$$
\underline{\operatorname{dens}}\left\{n \in \mathbb{N}: T^{n} x \in y+V^{\prime}\right\}>0 .
$$

If $T^{n} x \in y+V^{\prime}$, then for every $t \in[n, n+\delta]$

$$
T_{t} x-y=T_{t-n}\left(T_{n} x-y\right)+T_{t-n} y-y \in T_{t-n}\left(V^{\prime}\right)+V \subseteq V+V \subseteq U .
$$

Thus, for every $N \in \mathbb{N}$

hence

$$
\frac{\mu\left\{t \leq N: T_{t} x \in U\right\}}{N} \geq \delta \frac{\#\left\{n \in \mathbb{N}: n \leq N, T_{n} x \in y+V^{\prime}\right\}}{N},
$$

$\liminf _{N \rightarrow \infty} \frac{\mu\left\{t \leq N: T_{t} x \in U\right\}}{N} \geq \delta \liminf _{N \rightarrow \infty} \frac{\#\left\{n \in \mathbb{N}: n \leq N, T_{n} x \in y+V^{\prime}\right\}}{N}>0$.

Theorem 2.2. Let $\left(T_{t}\right)_{t \geq 0}$ be a $C_{0}$-semigroup on a separable Banach space $X$ such that there exist $X_{0} \subseteq X$, dense subset, and maps $S_{t}: X_{0} \rightarrow X, t>0$, satisfying:

(i) $T_{t} S_{t} x=x, T_{t} S_{r} x=S_{r-t} x$ for all $x \in X_{0}, t>0, r>t>0$;

(ii) $t \mapsto T_{t} x d t$ is Pettis integrable on $\left[0,+\infty\left[\right.\right.$ for all $x \in X_{0}$;

(iii) $t \mapsto S_{t} x d t$ is Pettis integrable on $\left[0,+\infty\left[\right.\right.$ for all $x \in X_{0}$.

Then $\left(T_{t}\right)_{t \geq 0}$ is frequently hypercyclic.

Proof. We will show that $T_{1}$ is a frequent hypercyclic operator. The assertion will follow by the previous result. First observe that for any $x \in X_{0}$, the map $t \mapsto S_{t} x$ is continuous; indeed, if we fix $r>t, S_{t} x=T_{r-t}\left(S_{r} x\right)$.

To verify that $T_{1}$ is frequently hypercyclic, we will follow the proof of Theorem 2.4 in [10], by considering suitable unconditionally convergent series of integrals.

W.l.o.g., we assume that $X_{0}=\left\{y_{1}, y_{2}, \ldots\right\}$ is a countable set. Conditions (ii), (iii) and Corollary 4.4 imply that there is an increasing sequence $\left\{N_{l}\right\}_{l \in \mathbb{N}}$ in $\mathbb{N}$ such that, for all $\lambda \leq l$ and all compact sets $K \subset\left[N_{l},+\infty[\right.$, we have

$$
\begin{gathered}
\left\|\int_{K} T_{t} y_{\lambda} d t\right\|<\frac{1}{l 2^{l}}, \\
\left\|\int_{K} S_{t} y_{\lambda} d t\right\|<\frac{1}{l 2^{l}} .
\end{gathered}
$$

For every $l, \nu \in \mathbb{N}$, set $\rho(l, \nu)=\nu$ and apply Lemma 2.5 in [11] to find pairwise disjoint sets $A(l, \nu) \subseteq \mathbb{N}, l, \nu \in \mathbb{N}$, of positive lower density such that, for all $n \in$ $A(l, \nu), m \in A(k, \nu)$, with $n \neq m$,

$$
n \geq \nu, \quad|n-m| \geq \nu+\mu .
$$

Define now

$$
z_{n}= \begin{cases}y_{l}, & n \in A\left(l, N_{l}\right) \\ 0, & \text { otherwise }\end{cases}
$$

and set 


$$
x:=\sum_{n \geq 1} \int_{n}^{n+1} S_{t} z_{n} d t .
$$

To see that this series is convergent, observe that, for each $l \in \mathbb{N}$,

$$
\sum_{n \in A\left(l, N_{l}\right)} \int_{n}^{n+1} S_{t} z_{n} d t=\sum_{n \in A\left(l, N_{l}\right)} \int_{n}^{n+1} S_{t} y_{l} d t
$$

converges unconditionally by (2.2). On the other hand, for every finite subset $F \subset$ $A\left(l, N_{l}\right)$, by (2.3) we have that $\bigcup_{n \in F}[n . n+1] \subset\left[N_{l},+\infty[\right.$, hence, by (2.3),

$$
\left\|\sum_{n \in F} \int_{n}^{n+1} S_{t} y_{l} d t\right\| \leq \frac{1}{l 2^{l}} .
$$

Therefore we easily obtain that the series in (2.5) is convergent.

Fix $l \in \mathbb{N}$ and $n \in A\left(l, N_{l}\right)$. Then

$$
\begin{aligned}
T_{n+1} x & = \\
= & \sum_{j<n} T_{n+1}\left(\int_{j}^{j+1} S_{t} z_{j} d t\right)+T_{n+1}\left(\int_{n}^{n+1} S_{t} z_{n} d t\right)+\sum_{j>n} T_{n+1}\left(\int_{j}^{j+1} S_{t} z_{j} d t\right) \\
= & \sum_{j<n} \int_{j}^{j+1} T_{n+1-t} z_{j} d t+\int_{n}^{n+1} T_{n+1-t} y_{l} d t+\sum_{j>n} \int_{j}^{j+1} S_{t-n-1} z_{j} d t \\
(2.8) & =\sum_{m<n} \int_{m}^{m+1} T_{s} z_{n-m} d s+u_{l}+\sum_{m=1}^{\infty} \int_{m-1}^{m} S_{r} z_{n+m} d r,
\end{aligned}
$$

where $u_{l}=\int_{0}^{1} T_{t} y_{l} d t$.

We analyze the first summand in (2.8):

$$
\begin{aligned}
& \sum_{m<n} \int_{m}^{m+1} T_{s} z_{n-m} d s= \\
= & \sum_{\lambda=1}^{l} \sum_{n-m \in A\left(\lambda, N_{\lambda}\right), m<n} \int_{m}^{m+1} T_{s} y_{\lambda} d s+\sum_{\lambda>1} \sum_{n-m \in A\left(\lambda, N_{\lambda}\right), m<n} \int_{m}^{m+1} T_{s} y_{\lambda} d s .
\end{aligned}
$$

By (2.3) , since $n \in A\left(l, N_{l}\right), n-m \in A\left(\lambda, N_{\lambda}\right)$, we get that $m=n-(n-m) \geq$ $N_{l}+N_{\lambda}$. Thus

$$
\left\|\sum_{m<n} \int_{m}^{m+1} T_{s} z_{n-m} d s\right\| \leq \sum_{\lambda=1}^{l} \frac{1}{l 2^{l}}+\sum_{\lambda>l} \frac{1}{\lambda 2^{\lambda}}<\frac{2}{2^{l}} .
$$

Analogously, by (2.2), we get

$$
\left\|\sum_{m=1}^{\infty} \int_{m-1}^{m} S_{r} z_{n+m} d r\right\|<\frac{2}{2^{l}}
$$

which gives, for every $n \in A\left(l, N_{l}\right)$ 


$$
\left\|T_{n+1} x-u_{l}\right\|<\frac{4}{2^{l}} .
$$

Since $A\left(l, N_{l}\right)$ has positive lower density for each $l \in \mathbb{N}$, we are done if we show that $\left(u_{l}\right)_{l}$ is a dense sequence in $X$. Indeed, $u_{l}=R y_{l}, l \in \mathbb{N}$, where $R$ is the continuous operator defined by

$$
R x:=\int_{0}^{1} T_{t} x d t
$$

We need to prove that $R$ has dense range. First observe that $I-T_{1}$ has dense range. Otherwise there would exists $\phi \in X^{\prime}, \phi \neq 0$, such that

$$
\left\langle\phi, x-T_{1} x\right\rangle=0 \text { for all } x \in X .
$$

This implies that for every $n \in \mathbb{N}$ and $x \in X$ :

$$
\langle\phi, x\rangle=\left\langle\phi, T_{n} x\right\rangle=0 \text { for all } x \in X .
$$

In particular, if $0<s$, then

$$
\int_{n}^{n+s}\left\langle\phi, T_{t} y_{l}\right\rangle>d t=\int_{0}^{s}\left\langle\phi, T_{u+n} y_{l}\right\rangle d u=\int_{0}^{s}\left\langle\phi, T_{u} y_{l}\right\rangle d u
$$

The left term tends to 0 , by (2.1), as $n \rightarrow \infty$. Since the right term is fixed and $s>0, l \in \mathbb{N}$ were arbitrary, we have

$$
\langle\phi, x\rangle=0 \text { for all } x \in X,
$$

which is a contradiction. Finally observe that if $(A, D(A))$ is the generator of $\left(T_{t}\right)_{t \geq 0}$, then for every $x \in D(A)$,

$$
\left(I-T_{1}\right) x=\int_{0}^{1} T_{t} A x=R(A x),
$$

thus $\left(I-T_{1}\right)(D(A)) \subseteq R(X)$. By the density of $D(A)$ in $X$, we get that

$$
X=\overline{\left(I-T_{1}\right)(X)}=\overline{\left(I-T_{1}\right)(D(A))} \subseteq \overline{R(X)}
$$

Corollary 2.3. Let $X$ be a separable complex Banach space, $\left(T_{t}\right)_{t \geq 0}$ a $C_{0}$-semigroup with generator A. Assume that there exists a family $\left(f_{j}\right)_{j \in \Gamma}$ of locally bounded measurable maps $f_{j}: I_{j} \rightarrow X$ such that $I_{j}$ is an interval in $\mathbb{R}, A f_{j}(t)=i t f_{j}(t)$ for every $t \in I_{j}, j \in \Gamma$ and $\operatorname{span}\left\{f_{j}(t): j \in \Gamma, t \in I_{j}\right\}$ is dense in $X$.

If either

a) $f_{j} \in C^{2}\left(I_{j}, X\right)$ for every $j \in \Gamma$

or

b) $X$ does not contain $c_{0}$ and for every $\varphi \in X^{\prime}$ and $j \in \Gamma$ the $\operatorname{map}\left\langle\varphi, f_{j}\right\rangle \in C^{1}\left(I_{j}\right)$, then $\left(T_{t}\right)_{t \geq 0}$ is frequently hypercyclic.

Proof. .

First we prove the following:

a)' if a) holds then there exists a family $\left(g_{j}\right)_{j \in \Lambda}$ of functions $g_{j} \in C^{2}(\mathbb{R}, X)$ with compact support such that $A g_{j}(t)=i t g_{j}(t)$ for every $t \in \mathbb{R}, j \in \Lambda$ and $\operatorname{span}\left\{g_{j}(t): j \in \Lambda, t \in \mathbb{R}\right\}$ is dense in $X$. 
b)' if b) holds then there exists a family $\left(g_{j}\right)_{j \in \Lambda}$ of bounded measurable functions $g_{j}: \mathbb{R} \rightarrow X$ with compact support such that $\left\langle\varphi, g_{j}\right\rangle \in C^{1}(\mathbb{R})$ for every $\varphi \in X^{\prime}$, $A g_{j}(t)=i t g_{j}(t)$ for every $t \in \mathbb{R}, j \in \Lambda$ and $\operatorname{span}\left\{g_{j}(t): j \in \Lambda, t \in \mathbb{R}\right\}$ is dense in $X$.

If $\left.I_{j}=\right] x_{j}-r_{j}, x_{j}+r_{j}\left[\right.$ is a bounded interval, consider a sequence $\left(\phi_{n}^{j}\right)_{n} \in C^{\infty}(\mathbb{R})$ such that $\phi_{n}^{j}(s)=1$ if $\left|s-x_{j}\right| \leq r-\frac{1}{2 n}$ and $\phi_{n}^{j}(s)=0$ if $\left|s-x_{j}\right|>r_{j}-\frac{1}{n}$. If we extend $f_{j}$ outside $I_{j}$ setting $f_{j}=0$ in $\mathbb{R} \backslash I_{j}$, we have that $\phi_{n}^{j} f \in C^{2}(\mathbb{R}, X)$ for every $n \in \mathbb{N}$ if a) holds and $\left\langle\varphi, \phi_{n}^{j} f_{j}\right\rangle \in C^{1}(\mathbb{R})$ for every $\varphi \in X^{\prime}$ if b) holds. Moreover $\left(\phi_{n}^{j} f_{j}\right)_{n}$ converges pointwise to $f_{j}$ and

$$
A\left(\phi_{n}^{j}(t) f_{j}(t)\right)=\phi_{n}^{j} A f_{j}(t)=i t \phi_{n}^{j} f_{j}(t)
$$

for every $t \in \mathbb{R}, j \in \Gamma$.

If the interval $I_{j}$ is unbounded, for example $\left.I_{j}=\right] a_{j},+\infty[$, the argument runs analogously, by considering functions $\phi_{n}^{j} \in C^{\infty}(\mathbb{R})$ with support in $] a_{j}+\frac{1}{n}, n[$.

It remains to show that

$$
\overline{\operatorname{span}\left\{\phi_{n}^{j} f_{j}(t): j \in \Gamma, t \in I_{j}, n \in \mathbb{N}\right\}}=X .
$$

If $\varphi \in X^{\prime}$ and $\left\langle\varphi, \phi_{n}^{j}(t) f_{j}(t)\right\rangle=0$ for every $j \in \Gamma, t \in I_{j}, n \in \mathbb{N}$, then, by taking the limit as $n \rightarrow \infty$, we get that $\left\langle\varphi, f_{j}(t)\right\rangle=0$ for every $t \in I_{j}$ and $j \in \Gamma$. Then, by the assumption on the ranges of the $f_{j}$, we get that $\varphi=0$.

¿From now on, let $\Lambda=\{(j, n): j \in \Gamma, n \in \mathbb{N}\}$ and, for every $\lambda=(j, n) \in \Lambda$, set $g_{\lambda}=\phi_{n}^{j} f_{j}$.

For every $r \in \mathbb{R}$ and $\lambda \in \Lambda$, set

$$
\psi_{r, \lambda}:=\int_{\mathbb{R}} e^{-i r s} g_{\lambda}(s) d s=\mathcal{F}\left(g_{\lambda}\right)(r),
$$

where $\mathcal{F}$ denotes the $X$-valued Fourier transform. The set $\left\{\psi_{r, \lambda}: r \in \mathbb{R}, \lambda \in \Lambda\right\}$ is dense in $X$. Indeed, let $\varphi \in X^{\prime}$ such that for all $r \in \mathbb{R}, \lambda \in \Lambda$,

$$
\left\langle\varphi, \psi_{r, \lambda}\right\rangle=\int_{\mathbb{R}} e^{-i r s}\left\langle\varphi, g_{\lambda}\right\rangle d s=0
$$

This means that the Fourier transform of the (scalar) function $s \mapsto\left\langle\varphi, g_{\lambda}\right\rangle$ vanishes on $\mathbb{R}$, hence, observing that $\left\langle\varphi, g_{\lambda}\right\rangle$ is continuous, we get that $\left\langle\varphi, g_{\lambda}\right\rangle=0$ on $\mathbb{R}$, hence $\varphi=0$.

For every $t>0$ set

It holds

$$
S_{t} \psi_{r, \lambda}:=\int_{\mathbb{R}} e^{-i(t+r) s} g_{\lambda}(s) d s=\mathcal{F}\left(g_{\lambda}\right)(t+r)=\psi_{t+r, \lambda}
$$

$$
T_{t} \psi_{r, \lambda}=\int_{\mathbb{R}} e^{i(t-r) s} g_{\lambda}(s) d s=\mathcal{F}\left(g_{\lambda}\right)(-t+r)=\psi_{-t+r, \lambda},
$$

and $T_{t} S_{t} \psi_{r, \lambda}=\psi_{r, \lambda}, T_{t} S_{s} \psi_{r, \lambda}=S_{s-t} \psi_{r, \lambda}$ for all $\lambda \in \Lambda, r \in \mathbb{R}, s>t>0$.

It remains to show that $t \mapsto S_{t} \psi_{r, \lambda} d t$ and $t \mapsto T_{t} \psi_{r, \lambda} d t$ are Pettis integrable on $[0,+\infty[$ for every $r \in \mathbb{R}$ and $\lambda \in \Lambda$.

In the case a)', $\mathcal{F}\left(g_{\lambda}\right)$ is Bochner integrable. Indeed, $g_{\lambda} \in C^{2}(\mathbb{R}, X)$ and has compact support. Hence $g_{\lambda}^{\prime \prime}$ is Fourier integrable and 


$$
\mathcal{F}\left(g_{\lambda}^{\prime \prime}\right)(r)=-r^{2} \mathcal{F}\left(g_{\lambda}\right)
$$

Therefore $\mathcal{F}\left(g_{\lambda}\right)$ is Bochner integrable on $\mathbb{R}$. It follows that $t \mapsto T_{t}\left(\psi_{r, \lambda}\right)$ and $t \mapsto S_{t}\left(\psi_{r, \lambda}\right)$ are Bochner integrable on $[0,+\infty[$.

In the case $b$ )', we prove that $\mathcal{F}\left(g_{\lambda}\right)$ is Pettis integrable on $[0,+\infty[$. It will follow

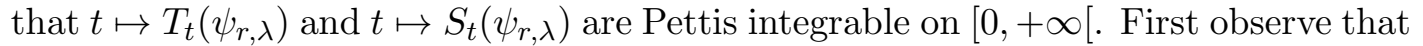
$\mathcal{F}\left(g_{\lambda}\right)$ is continuous, hence measurable. Let $\varphi \in X^{\prime}$ and consider $g(s)=\left\langle\varphi, g_{\lambda}(s)\right\rangle \in$ $C_{c}^{1}(\mathbb{R})$. We have:

$$
\left\langle\varphi, \mathcal{F}\left(g_{\lambda}\right)(r)\right\rangle=\int_{\mathbb{R}} e^{-i r s}\left\langle\varphi, g_{\lambda}(s)\right\rangle d s=\mathcal{F}(g)(r) .
$$

We have that $g^{\prime} \in L^{2}(\mathbb{R}) \cap L^{1}(\mathbb{R})$ and $\mathcal{F}\left(g^{\prime}\right)(r)=i r \mathcal{F}(g)(r) \in L^{2}(\mathbb{R})$. Hence, for $a>0$ :

$$
\int_{|r|>a}|\mathcal{F}(g)(r)| d r \leq\left(\int_{|r| \geq a} \frac{1}{r^{2}} d r\right)^{\frac{1}{2}}\left(\int_{|r|>a} r^{2}|\mathcal{F}(g)|^{2}\right)^{\frac{1}{2}}<+\infty .
$$

Therefore $\mathcal{F}(g) \in L^{1}(\mathbb{R})$. By Theorem 4.5, this implies that $\mathcal{F}\left(g_{\lambda}\right)$ is Pettis integrable on $[0,+\infty[$.

Remark 2.4. With the same argument of [23, Remark 2.2], one can show that the Desch-Schappacher-Webb criterion for chaoticity of $C_{0}$-semigroups (see [21]) implies frequent hypercyclicity.

Remark 2.5. There is a connection between Corollary 2.3 and the recent results of S. Grivaux in [26]. Indeed assume that one of the conditions a) or b) (or equivalently a)' or b)') hold for a countable family of locally bounded functions $\left\{f_{j}\right\}_{j \in \mathbb{N}}$. Define for every $j, k \in \mathbb{N}, \theta \in[0,2 \pi[$

$$
E_{j, k}\left(e^{i \theta}\right)=f_{j}(\theta+2 k \pi) .
$$

The family $\left\{E_{j, k}: j, k \in \mathbb{N}\right\}$ is a countable family of bounded $\mathbb{T}$-eigenvectors fields for the operator $T_{1}$, where $\mathbb{T}=\{\lambda \in \mathbb{C}:|\lambda|=1\}$, such that $\operatorname{span}\left\{E_{j, k}(\lambda): \lambda \in\right.$ $\mathbb{T}, j, k \in \mathbb{N}\}$ is dense in $X$. Actually it holds that $\operatorname{span}\left\{E_{j, k}(\lambda): \lambda \in \mathbb{T} \backslash D, j, k \in \mathbb{N}\right\}$ is dense in $X$ for every countable subset of $\left(T_{t}\right)_{t \geq 0}$. Indeed, if $D=\left\{e^{i \theta_{n}}: n \in \mathbb{N}\right\}$, with $\theta_{n} \in[0,2 \pi[$, then

$\operatorname{span}\left\{E_{j, k}(\lambda): \lambda \in \mathbb{T} \backslash D j, k \in \mathbb{N}\right\}=\operatorname{span}\left\{f_{j}(s): s \in \mathbb{R} \backslash\left\{\theta_{n}+2 k \pi: k \in \mathbb{N}\right\}, j \in \mathbb{N}\right\}$.

By the weak continuity of the vector field $f_{j}$, we get that the right hand set is dense in $X$.

Thus, by [26, Proposition 4.2] $T_{1}$ has perfectly spanning eigenvectors associated to unimodular eigenvectors, i.e. there exists a probability measure $\sigma$ on the unit circle $\mathbb{T}$ such that for every $\sigma$-measurable subset of $A$ with $\sigma(A)=1, \operatorname{span}\{\operatorname{ker}(T-\lambda)$ : $\lambda \in A\}$ is dense in $X$.

Example 2.6. Consider the linear perturbation of the one-dimensional OrnsteinUhlenbeck operator

$$
\mathcal{A}_{\alpha} u=u^{\prime \prime}+b x u^{\prime}+\alpha u
$$


where $\alpha \in \mathbb{R}$, with domain

$$
D\left(\mathcal{A}_{\alpha}\right)=\left\{u \in L^{2}(\mathbb{R}) \cap W_{\text {loc }}^{2,2}(\mathbb{R}): \mathcal{A}_{\alpha} u \in L^{2}(\mathbb{R})\right\} .
$$

In [14, it was proved that if $\alpha>b / 2>0$, then the semigroup generated by $\mathcal{A}_{\alpha}$ in $L^{2}(\mathbb{R})$ is chaotic. Actually the semigroup is frequently hypercyclic.

Indeed, for every $\mu \in \mathbb{C}$, with $\Re \mu<-\frac{b}{2}+\alpha$ the functions $u_{\mu}^{1}$ and $u_{\mu}^{2}$, whose Fourier transforms are

$$
\widehat{u_{\mu}^{1}}(\xi)=e^{-\xi^{2} / 2 b} \xi|\xi|^{-(2+(\mu-\alpha) / b)}, \quad \widehat{u_{\mu}^{2}}(\xi)=e^{-\xi^{2} / 2 b}|\xi|^{-(1+(\mu-\alpha) / b)},
$$

are eigenfunctions of $\mathcal{A}_{\alpha}$ (see [14, 33]).

For every $s \in \mathbb{R}$, consider the functions $f_{1}(s)=u_{i s}^{1}$ and $f_{2}(s)=u_{i s}^{2}$. For every $\phi \in X^{\prime}=L^{2}(\mathbb{R})$ and $j=1,2$, by Parseval equality, we have

$$
\left\langle\phi, f_{j}(s)\right\rangle=\int_{\mathbb{R}} \phi(x) u_{i s}^{j}(x) d x=\int_{\mathbb{R}} \widehat{\phi}(x) \widehat{u}_{i s}^{j}(x) d x \quad s \in \mathbb{R} .
$$

It is immediate to verify that $\left\langle\phi, f_{j}\right\rangle \in C^{1}(\mathbb{R})$, by Lebesgue's theorem.

The argument of [14] shows that $\operatorname{span}\left\{f_{i}(s): i=1,2, s \in \mathbb{R}\right\}$ is dense in $L^{2}(\mathbb{R})$. Therefore the semigroup is frequently hypercyclic by Corollary 2.3 .

We will see that the Frequent Hypercyclicity Criterion for semigroups implies chaos for each single operator of the semigroup. It is interesting to observe that this is in general stronger than the chaoticity of the semigroup since, by recent results of Bayart and Bermúdez [5], there are chaotic $C_{0}$-semigroups $\left(T_{t}\right)_{t \geq 0}$ such that no single operator $T_{t}$ is chaotic, and chaotic $C_{0}$-semigroups $\left(T_{t}\right)_{t \geq 0}$ containing non-chaotic operators $T_{t_{0}}$, $t_{0}>0$, and at the same time chaotic $T_{t_{1}}$ for some $t_{1}>0$.

Proposition 2.7. Let $X$ be a separable Banach space and let $\left(T_{t}\right)_{t \geq 0}$ be a $C_{0}$ semigroup on $X$ that satisfies the Frequent Hypercyclicity Criterion of Theorem 2.2. Then the operator $T_{t_{0}}$ is chaotic for every $t_{0}>0$.

Proof. Given $t_{0}>0$, we know that $T_{t_{0}}$ is frequently hypercyclic, thus hypercyclic [15. Given $x \in X$ and $\varepsilon>0$ we want to find a $T_{t_{0}}$-periodic point $z \in X$ such that $\|x-z\|<\varepsilon$. Indeed, let $y \in X_{0}$ such that $\|x-y\|<\varepsilon$. By continuity, we fix $\delta>0$ such that

$$
\left\|x-\delta^{-1} \int_{0}^{\delta} T_{s} y d s\right\|<\varepsilon .
$$

Now, let $n \in \mathbb{N}$ big enough so that, by Corollary 4.4 and for $t:=n t_{0}$, we have that

$$
z:=\delta^{-1}\left[\sum_{k \geq 1} \int_{0}^{\delta} S_{k t-s} y d s+\int_{0}^{\delta} T_{s} y d s+\sum_{k \geq 1} \int_{0}^{\delta} T_{k t+s} y d s\right]
$$

satisfies $\|x-z\|<\varepsilon$. Finally, observe that the hypothesis of the Frequent Hypercyclicity Criterion and continuity give $T_{t_{0}}^{n} z=T_{t} z=z$.

Finally we point out the connection between the Frequent Hypercyclicity Criterion for semigroups and the Frequent Hypercyclicity Criterion for sequences of operators.

Proposition 2.8. Let $X$ be a separable Banach space and let $\left(T_{t}\right)_{t \geq 0}$ be a $C_{0}$ semigroup on $X$. If there exists $X_{0} \subseteq X$, dense subset, with $T_{t}\left(X_{0}\right) \subseteq X_{0}$ for every $t>0$, and maps $S_{t}: X_{0} \rightarrow X_{0}, t>0$, satisfying: 
(i) $T_{t} S_{t} x=x, S_{r} T_{t} x=T_{t} S_{r} x=S_{r-t} x$ for all $x \in X_{0}, t>0, r>t>0$;

(ii) $t \mapsto T_{t} x d t$ is Pettis integrable on $\left[0,+\infty\left[\right.\right.$ for all $x \in X_{0}$;

(iii) $t \mapsto S_{t} x d t$ is Pettis integrable on $\left[0,+\infty\left[\right.\right.$ for all $x \in X_{0}$.

then the operator $T_{t_{0}}$ satisfies the Frequent Hypercyclicity criterion for operators for every $t_{0}>0$.

Proof. By sake of simplicity, let $t_{0}=1$. First observe that $S_{n} x=S_{n} T_{1} S_{1} x=$ $S_{n-1} S_{1} x=S_{n-2} S_{1}^{2}=\ldots=S_{1}^{n} x$ for every $x \in X_{0}$. For every $x \in X_{0}$, set $y=\int_{0}^{1} T_{t} x d t$. Then the series

$$
\sum_{n=1}^{\infty} T_{n} y=\sum_{n=1}^{\infty} \int_{n}^{n+1} T_{t} x d t
$$

is unconditionally convergent by Proposition 4.3. Analogously, since

$$
\int_{n-1}^{n} S_{s} x d s=\int_{0}^{1} S_{n-1+s} x d s=\int_{0}^{1} S_{n-u} x d s=S_{n} \int_{0}^{1} T_{s} x d s=S_{n} y
$$

we get that the series $\sum_{n=1}^{\infty} S_{n} y$ is unconditionally convergent. Finally we observe that, by the same argument used in the proof of Theorem 2.2, the set $\left\{\int_{0}^{1} T_{t} x: x \in\right.$ $\left.X_{0}\right\}$ is dense.

Given a $C_{0}$-semigroup $\left(T_{t}\right)_{t \geq 0}$, it seems a reasonable guess that if $T_{t_{0}}$ satisfies the Frequent Hypercyclicity Criterion for operators of Proposition 1.1 for some $t_{0}>0$, then the semigroup $\left(T_{t}\right)_{t \geq 0}$ should satisfy the Frequent Hypercyclicity Criterion for semigroups of Theorem 2.2. Unfortunately, we do not know whether this holds in general.

\section{Translation Semigroups}

An admissible weight function on $[0,+\infty[$ is a measurable function $\rho:[0,+\infty[\rightarrow \mathbb{R}$ satisfying the following conditions:

(i) $\rho(t)>0$ for all $t \in[0,+\infty[$;

(ii) there exist constants $M \geq 1$ and $\omega \in \mathbb{R}$ such that $\rho(\tau) \leq M e^{\omega t} \rho(\tau+t)$ for all $\tau \in[0,+\infty[$ and all $t>0$.

We recall the following useful results:

Lemma 3.1. If $\rho$ is an admissible weight, then for every $l>0$ there exist $A, B>0$ such that for every $\sigma \in[0,+\infty[$ and for every $t \in[\sigma, \sigma+l]$, it holds

$$
A \rho(\sigma) \leq \rho(t) \leq B \rho(\sigma+l) \text {. }
$$

Lemma 3.2. Let $\rho:\left[0,+\infty\left[\rightarrow \mathbb{R}^{+}\right.\right.$be an admissible weight.

(1) The following conditions are equivalent:

(i) For all $b \geq 0$ the series $\sum_{k=1}^{\infty} \rho(b+k)$ is convergent,

(ii) For all $b \geq 0$ there exists $P>0$ such that the series $\sum_{k=1}^{\infty} \rho(b+k P)$ is convergent,

(iii) There exists $P>0$ such that the series $\sum_{k=1}^{\infty} \rho(k P)$ is convergent,

(iv) The series $\sum_{k=1}^{\infty} \rho(k)$ is convergent,

(v) $\int_{0}^{+\infty} \rho(s) d s<+\infty$,

(vi) there exists $D \subseteq \mathbb{N}$ with bounded gaps (i.e. there exists $M>0$ such that $D \cap[n, n+M] \neq \emptyset$ for every $n \in \mathbb{N})$ such that $\sum_{k \in D} \rho(k)$ is convergent. 
(2) $\rho$ is bounded if and only if there exists $D \subseteq \mathbb{N}$ with bounded gaps such that $\rho$ is bounded on $D$.

Proof. 1. The implications (i) $\Rightarrow$ (ii) and (ii) $\Rightarrow$ (iii) are obvious. If (iii) holds, we fix $n \in \mathbb{N}$ with $n>P$, and $A, B>0$ satisfying the inequalities of Lemma 3.1 for $l=P$. For all $k \in \mathbb{N}$ there exists $m_{k} \in \mathbb{N}$ such that $k n \in\left[\left(m_{k}-1\right) P, m_{k} P\right]$. Since $n>P$ then $m_{k+1}>m_{k}$ for all $k \in \mathbb{N}$. Thus,

$$
\sum_{k=1}^{\infty} \rho(k n) \leq B \sum_{k=1}^{\infty} \rho\left(m_{k} P\right) \leq B \sum_{k=1}^{\infty} \rho(k P)<+\infty .
$$

Analogously, $\sum_{k=1}^{\infty} \rho(k n+j) \leq B \sum_{k=1}^{\infty} \rho(k P), j=1, \ldots, n-1$, and we obtain that $\sum_{k=1}^{\infty} \rho(k)<+\infty$.

If (iv) holds, then

$$
\int_{0}^{+\infty} \rho(s) d s=\sum_{k=1}^{\infty} \int_{k-1}^{k} \rho(s) d s \leq B \sum_{k=1}^{\infty} \rho(k)<+\infty
$$

by an application of Lemma 3.1 for $l=1$, which yields (v).

If $\int_{0}^{+\infty} \rho(s) d s<+\infty$ and $b \geq 0$ is arbitrary, then

$$
\sum_{k=1}^{\infty} \rho(b+k) \leq A^{-1} \sum_{k=1}^{\infty} \int_{b+k}^{b+k+1} \rho(s) d s=A^{-1} \int_{b+1}^{+\infty} \rho(s) d s<+\infty,
$$

where we applied again Lemma 3.1 for $l=1$. This concludes that (v) implies (i).

Finally, clearly (iii) implies (vi). We prove that (vi) implies (iii). Assume w.l.o.g. that $M \in \mathbb{N}$. Then for every $k \in \mathbb{N}$ there exists $h_{k} \in D \cap[M k, M(k+1)]$. If $A, B>0$ satisfy the inequalities of Lemma 3.1 for $l=M$, we have that

$$
A \rho(M k) \leq \rho\left(h_{k}\right) \leq B \rho(M(k+1)), \quad k \in \mathbb{N}
$$

Thus

$$
\sum_{k=1}^{\infty} \rho(M k) \leq A^{-1} \sum_{k=1}^{\infty} \rho\left(h_{k}\right) \leq A^{-1} \sum_{h \in D} \rho(h)<+\infty .
$$

2. Assume that $\rho(h) \leq K$ for every $h \in D$, where $D \subseteq \mathbb{N}$ with bounded gaps. Hence, there is $M \in \mathbb{N}$ such that $[M n, M n+M] \cap D \neq \emptyset$ for every $n \in \mathbb{N}$. Choose $h_{n} \in[M n, M n+M] \cap D$. By Lemma 3.1, there exists $A_{M}, B_{M}>0$ such that

$$
A_{M} \rho(M n) \leq \rho\left(h_{n}\right) \leq B_{M} \rho(M n+M), \quad n \in \mathbb{N},
$$

hence $\rho(M n) \leq A_{M}^{-1} K$ for every $n \in \mathbb{N}$. On the other hand, for every $s \geq 0$ there exists $\bar{n}$ such that $x \in[M \bar{n}, M \bar{n}+M]$,and therefore

$$
\rho(s) \leq B_{M} \rho(M \bar{n}+M) \leq K A_{M}^{-1} B_{M}
$$

Next we consider the following function spaces:

$$
L_{p}^{\rho}\left(\left[0,+\infty[)=\left\{u:\left[0,+\infty\left[\rightarrow \mathbb{R}: u \text { is measurable and }\|u\|_{p}<\infty\right\},\right.\right.\right.\right.
$$

where $\|u\|_{p}=\left(\int_{0}^{\infty}|u(t)|^{p} \rho(t) d t\right)^{\frac{1}{p}}$, and 


$$
C_{0}^{\rho}\left(\left[0,+\infty[)=\left\{u:\left[0,+\infty\left[\rightarrow \mathbb{R}: u \text { is continuous and } \lim _{x \rightarrow \infty} u(x) \rho(x)=0\right\},\right.\right.\right.\right.
$$

with $\|u\|_{\infty}=\sup _{t \in[0,+\infty[}|u(t)| \rho(t)$.

If $X$ is any of the spaces above, the translation semigroup $\left(T_{t}\right)_{t \geq 0}$ is defined as usual by

$$
T_{t} f(x)=f(x+t), \quad t \geq 0, x \in I,
$$

and is a $C_{0}$-semigroup (see e.g. [21]).

Hypercyclic and chaotic translation semigroups have been characterized in [21, 19, 32. If $X$ is one of the spaces $L_{p}^{\rho}\left(\left[0,+\infty[)\right.\right.$ or $C_{0}^{\rho}([0,+\infty[)$ with an admissible weight function $\rho$, the translation semigroup $\left(T_{t}\right)_{t \geq 0}$ on $X$ is hypercyclic if and only if $\liminf _{t \rightarrow+\infty} \rho(t)=0$.

If $X=C_{0}^{\rho}\left(\left[0,+\infty[)\right.\right.$, then the translation semigroup $\left(T_{t}\right)_{t \geq 0}$ on $X$ is chaotic if and only if $\lim _{x \rightarrow+\infty} \rho(x)=0$.

If $X=L_{p}^{\rho}\left(\left[0,+\infty[),\left(T_{t}\right)_{t \geq 0}\right.\right.$ is chaotic if and only if any of the conditions of lemma 3.2 is satisfied.

Proposition 3.3. Let $\rho$ be an admissible weight on $\left[0,+\infty\left[, X=L_{\rho}^{p}([0,+\infty[), 1 \leq\right.\right.$ $p<+\infty$ and $\left(T_{t}\right)_{t \geq 0}$ the translation semigroup on $X$. Then $\left(T_{t}\right)_{t \geq 0}$ is chaotic if and only if it satisfies the Frequent Hypercyclicity Criterion for semigroups.

Proof. Let $X=L_{\rho}^{p}\left(\left[0,+\infty[)\right.\right.$. If $\left(T_{t}\right)_{t \geq 0}$ is chaotic, then $\int_{0}^{+\infty} \rho(s) d s$ is finite. Let $X_{0}$ be the space generated by the characteristic functions of bounded intervals of $[0,+\infty[$. $X_{0}$ is dense in $L_{\rho}^{p}\left(\left[0,+\infty[)\right.\right.$. For every $t>0$ and $f \in X_{0}$ set

$$
S_{t} f(s)= \begin{cases}f(s-t) & s \geq t \\ 0 & s \in[0, t[\end{cases}
$$

Observe that $T_{t} S_{t} f=f$ and $T_{t} S_{r} f=S_{r-t} f$ for all $f \in X_{0}, t>0, r>t>0$. Moreover $\int_{\mathbb{R}^{+}}\left\|T_{t} f\right\| d t$ converges for all $f \in X_{0}$, because of the compact support of $f$, hence $\int_{\mathbb{R}^{+}} T_{t} f$ is Pettis integrable. On the other hand, consider $f=\chi_{[a, b]}$, with $0 \leq a<b$. If $p=1$, we have

$$
\left\|S_{t} f\right\|=\int_{t}^{t+b} \rho(s) d s=\int_{0}^{b} \rho(s+t) d s \leq b B \rho(t+b)
$$

where $B$ is a positive costants such that for every $s \in[0, b]$ and for every $t \geq 0$

$$
\rho(s+t) \leq B \rho(t+b) .
$$

Since $\int_{0}^{\infty} \rho(t+b) d t$ is finite, we get that $t \mapsto S_{t} f$ is Pettis integrable. Let $p>1$ and let $\phi \in L_{\rho}^{p^{\prime}}\left(\left[0,+\infty[)\right.\right.$, where $\frac{1}{p}+\frac{1}{p^{\prime}}=1$. To prove that $t \mapsto S_{t} f$ is Pettis integrable, by Theorem 4.5, we have to show that $t \mapsto\left\langle\phi, S_{t} f\right\rangle \in L^{1}([0,+\infty[)$. It holds that

$$
\left\langle\phi, S_{t} f\right\rangle=\int_{t}^{+\infty} f(s-t) \rho(s) d s=\int_{0}^{+\infty} f(u) \rho(t+u) d u .
$$

A straightforward application of Tonelli and Fubini theorems (as for the proof of the integrability of the convolution) gives the assertion.

Proposition 3.4. Let $\rho$ be an admissible weight on $\left[0,+\infty\left[\right.\right.$ and $\left(T_{t}\right)_{t \geq 0}$ the translation semigroup on $C_{0}^{\rho}\left(\left[0,+\infty[)\right.\right.$. If $\int_{0}^{+\infty} \rho(s) d s<+\infty$, then $\left(T_{t}\right)_{t \geq 0}$ satisfies the frequent hypercyclicity criterion for semigroups. 
Proof. Let $X_{0}$ be the space of continuous functions on $[0,+\infty$ [ with compact support. For every $t>0$ and each $f \in X_{0}$ set

$$
S_{t} f(s)= \begin{cases}f(s-t) & s \geq t \\ f(0) & s \in[0, t[\end{cases}
$$

Observe that $T_{t} S_{t} f=f$ and $T_{t} S_{r} f=S_{r-t} f$ for all $f \in X_{0}, t>0, r>t>0$. Moreover, because of the compact support of $f, T_{t} f=0$ for $t$ big enough. Hence $\int_{0}^{+\infty}\left\|T_{t} f\right\| d t$ converges for all $f \in X_{0}$. On the other hand, if $f \in X_{0}$ and supp $f \subseteq$ $[0, b]$, then

$$
\left\|S_{t} f\right\|=\sup _{s \in[t, t+b]}|f(s-t)| \rho(s)=\sup _{s \in[0, b]}|f(s)| \rho(s+t) \leq B A^{-1} \rho(0)^{-1} \rho(t+b)\|f\|,
$$

where $A, B$ are positive constants such that for every $s \in[0, b]$ and for every $t \geq 0$

$$
A \rho(t) \leq \rho(s+t) \leq B \rho(t+b) .
$$

Since $\int_{0}^{+\infty} \rho(t+b) d t$ is finite, we get that $\int_{0}^{+\infty} S_{t} f d t$ converges unconditionally.

Remark 3.5. It should be observed that, for the translation semigroup $\left(T_{t}\right)_{t \geq 0}$ on $L_{p}^{\rho}\left(\left[0,+\infty[)\right.\right.$, it holds that $\left(T_{t}\right)_{t \geq 0}$ is chaotic if and only if every operator $T_{t}$ satisfies the Frequent Hypercyclicity Criterion for operators by Proposition 2.8.

In [27] the authors obtain a necessary condition for frequent hypercyclicity of unilateral weighted shifts on $\ell^{p}$. Inspired by their condition, we obtain an analogous one for translation semigroups.

Proposition 3.6. Let $\rho$ be an admissible weight on $\left[0,+\infty\left[,\left(T_{t}\right)_{t \geq 0}\right.\right.$ the translation semigroup in $L_{p}^{\rho}\left(\left[0,+\infty[)\right.\right.$. If $\left(T_{t}\right)_{t \geq 0}$ is frequently hypercyclic, then for every $\varepsilon>0$ there exists a sequence $\left(n_{k}\right)_{k}$ in $\mathbb{N}$ with positive lower density such that

$$
\sum_{k>i} \rho\left(n_{k}-n_{i}\right)<\varepsilon
$$

Moreover, $\rho$ is bounded.

Proof. By the results in [15], the operator $T_{1}$ is frequently hypercyclic. Let $f \in$ $L_{p}^{\rho}\left(\left[0,+\infty[)\right.\right.$ be frequently hypercyclic for $T_{1}$. If $u$ is the characteristic function of $[0,1], \rho_{0}=\left(\int_{0}^{1} \rho(s) d s\right)^{-\frac{1}{p}}$, and $0<\eta<1$, there exists a sequence $\left(n_{k}\right)_{k}$ in $\mathbb{N}$ with positive lower density such that $\left\|T_{n_{k}} f-\rho_{0} u\right\|<\eta, k \in \mathbb{N}$. This implies that

hence

$$
\left(\int_{0}^{1}\left|f\left(s+n_{k}\right)-\rho_{0}\right|^{p} \rho(s) d s\right)^{\frac{1}{p}}<\eta,
$$

$$
\begin{aligned}
& 1=\rho_{0}\left(\int_{0}^{1} \rho(s) d s\right)^{\frac{1}{p}} \leq \\
\leq & \left(\int_{0}^{1}\left|f\left(s+n_{k}\right)-\rho_{0}\right|^{p} \rho(s) d s\right)^{\frac{1}{p}}+\left(\int_{0}^{1}\left|f\left(s+n_{k}\right)\right|^{p} \rho(s) d s\right)^{\frac{1}{p}}< \\
< & \eta+\left(\int_{0}^{1}\left|f\left(s+n_{k}\right)\right|^{p} \rho(s) d s\right)^{\frac{1}{p}} .
\end{aligned}
$$


So we get that

$$
\int_{0}^{1}\left|f\left(s+n_{k}\right)\right|^{p} \rho(s) d s>(1-\eta)^{p} .
$$

By lemma 3.1, there exist $A, B>0$ such that $A \rho(\tau) \leq \rho(s) \leq B \rho(\tau+1)$ for every $\tau \geq 0$ and $s \in[\tau, \tau+1]$. Hence

$$
\int_{0}^{1}\left|f\left(s+n_{k}\right)\right|^{p} d s \geq \frac{1}{B \rho(1)} \int_{0}^{1}\left|f\left(s+n_{k}\right)\right|^{p} \rho(s) d s>\frac{1}{B \rho(1)}(1-\eta)^{p}, \quad k \in \mathbb{N} .
$$

For all $i \in \mathbb{N}$ we have

$$
\begin{aligned}
& \eta^{p}>\int_{0}^{+\infty}\left|f\left(s+n_{i}\right)-\rho_{0} u(s)\right|^{p} \rho(s) d s>\int_{1}^{+\infty}\left|f\left(s+n_{i}\right)\right|^{p} \rho(s) d s \geq \\
\geq & \int_{n_{i}+1}^{\infty}|f(s)|^{p} \rho\left(s-n_{i}\right) d s=\sum_{j=1}^{\infty} \int_{n_{i}+j}^{n_{i}+j+1}|f(s)|^{p} \rho\left(s-n_{i}\right) d s \geq \\
\geq & \sum_{j>i}^{\infty} \int_{n_{j}}^{n_{j}+1}|f(s)|^{p} \rho\left(s-n_{i}\right) d s= \\
= & \sum_{j>i} \int_{0}^{1}\left|f\left(s+n_{j}\right)\right|^{p} \rho\left(s+n_{j}-n_{i}\right) d s \geq \\
\geq & A \sum_{j>i} \rho\left(n_{j}-n_{i}\right) \int_{0}^{1}\left|f\left(s+n_{j}\right)\right|^{p} d s>\frac{A(1-\eta)^{p}}{B \rho(1)} \sum_{j>i} \rho\left(n_{j}-n_{i}\right) .
\end{aligned}
$$

Hence

$$
\sum_{j>i} \rho\left(n_{j}-n_{i}\right)<\frac{B \rho(1)}{A(1-\eta)^{p}} \eta^{p} .
$$

Since $\eta \in] 0,1$ [ was arbitrary, we get the desired inequality. As a consequence of (3.1), since for every $A \subset \mathbb{N}$ with positive lower density the difference set $A-A$ has bounded gaps (see [35]), we have that there exists $D \subseteq \mathbb{N}$ with bounded gaps such that $\rho(h)<1$ for every $h \in D$. Hence $\rho$ is bounded by Lemma 3.2(2).

Example 3.7. Let $\phi: \mathbb{R}_{+} \rightarrow \mathbb{R}$ be a $C^{1}$-function with derivative bounded by $\omega>0$ and such that

$$
\limsup _{t \rightarrow+\infty} \phi(s)=+\infty, \liminf _{t \rightarrow-\infty} \phi(s)=-\infty .
$$

(For example, consider a $C_{1}$ function such that $\phi(s)=s \sin (\log s)$ if $s \geq 1$.) Set $\rho=e^{-\phi}$. Clearly $\rho>0$ and if $t, \tau>0$ we have

$$
\frac{\rho(\tau)}{\rho(t+\tau)}=e^{-\int_{\tau}^{t+\tau} \phi^{\prime}(s) d s} \leq e^{\omega \tau} .
$$

Hence $\rho$ is an admissible weight. The translation semigroup in $L_{p}^{\rho}([0,+\infty[)$ is hypercyclic, since $\liminf _{s \rightarrow \infty} \rho(s)=0$, but it is not frequently hypercyclic, since $\rho$ is unbounded. 
With a similar proof we get a necessary condition for frequently hypercyclic translation semigroups in weighted spaces of continuous functions.

Proposition 3.8. Let $\rho$ be an admissible weight on $\left[0,+\infty\left[\right.\right.$, and $\left(T_{t}\right)_{t \geq 0}$ the translation semigroup in $C_{0}^{\rho}\left(\left[0,+\infty[)\right.\right.$. If $\left(T_{t}\right)_{t \geq 0}$ is frequently hypercyclic, then for every $\varepsilon>0$ there exists a sequence $\left(n_{k}\right)_{k}$ in $\mathbb{N}$ with positive lower density such that $\rho\left(n_{k}-n_{i}\right)<\varepsilon$ for every $k>i$.

\section{Appendix}

We recall in this appendix the main definitions and results about Pettis integrability. Let $X$ be a Banach space and $(\Omega, \mu)$ a $\sigma$-finite measure space. A function $f: \Omega \rightarrow X$ is said to be weakly $\mu$-measurable if the scalar function $\varphi \circ f$ is $\mu$ measurable for every $\varphi \in X^{\prime}$, where $X^{\prime}$ denotes the topological dual of $X ; f$ is said to be $\mu$-measurable if there exists a sequence $\left(f_{n}\right)_{n}$ of simple functions such that $\lim _{n \rightarrow \infty}\left|f_{n}-f\right|=0 \mu$-a.e.

Lemma 4.1 (Dunford). If $f$ is weakly $\mu$-measurable and $\varphi \circ f \in L^{1}(\Omega, \mu)$ for every $\varphi \in X^{\prime}$, then for every measurable $E \subseteq \Omega$ there exists $x_{E} \in X^{\prime \prime}$ such that

$$
x_{E}(\varphi)=\int_{E} \varphi \circ f d \mu
$$

for every $\varphi \in X^{\prime}$.

Definition 4.2. If $f: \Omega \rightarrow X$ is weakly $\mu$-measurable and and $\varphi \circ f \in L^{1}(\Omega, \mu)$ for every $\varphi \in X^{\prime}$, then $f$ is called Dunford integrable. The Dunford integral of $f$ over a measurable $E \subseteq \Omega$ is defined by the element $x_{E} \in X^{\prime \prime}$ such that

$$
x_{E}(\varphi)=\int_{E} \varphi \circ f d \mu
$$

for every $\varphi \in X^{\prime}$.

In the case that $x_{E} \in X$ for every measurable $E$, then $f$ is called Pettis integrable and $x_{E}$ is called the Pettis integral of $f$ over $E$ and will be denoted by $(P)-\int_{E} f d \mu$.

Clearly the Dunford and Pettis integrals coincide if $X$ is a reflexive space.

Moreover, if $\|f\|$ is integrable on $\Omega$ (i.e. $f$ is Bochner integrable on $\Omega$ ), then $f$ is Pettis integrable on $X$.

Theorem 4.3 (Pettis). If $f$ is Pettis integrable, then for every sequence $\left(E_{n}\right)_{n}$ of disjoint measurable sets in $\Omega$

$$
\int_{\bigcup_{n \in \mathbb{N}} E_{n}} f d \mu=\sum_{n \in \mathbb{N}} \int_{E_{n}} f d \mu,
$$

where the series converges unconditionally.

Corollary 4.4. If $f:[0,+\infty[\rightarrow X$ is Pettis integrable on $[0,+\infty[$, then for every $\varepsilon>0$ there exists $N>0$ such that for every compact set $K \subset[N,+\infty[$

$$
\left\|\int_{K} f(t) d t\right\|<\varepsilon
$$


Proof. Assume that there exists $\varepsilon>0$ such that for every $n \in \mathbb{N}$ there exists a compact set $K_{n} \subseteq[n,+\infty[$ such that

$$
\left\|\int_{K_{n}} f(s) d s\right\|>\varepsilon
$$

It is easy to find a sequence $\left(k_{n}\right)_{n}$ of natural numbers such that the sets $K_{k_{n}}$ are mutually disjoint. Then

$$
\int_{\bigcup_{n} K_{k_{n}}} f(s) d s=\sum_{n=1}^{\infty} \int_{K_{n}} f(s) d s,
$$

hence $\lim _{n \rightarrow \infty} \int_{K_{n}} f(s) d s=0$, a contradiction.

Theorem 4.5. If the Banach space $X$ does not contain $c_{0}$ and $(\Omega, \mu)$ is $\sigma$-finite measure space, then a measurable Dunford integrable function $f: \Omega \rightarrow X$ is Pettis integrable.

The proofs of all these results can be found in [22] for the case of finite measure space, but they easily extend to $\sigma$-finite measure spaces. In particular, the proof of the deep Theorem 4.5 follows analogously to the finite measure space case ([22], Theorem 7, p.54) taking into account the following decomposition theorem due to J. K. Brooks (see [12], 2. Theorem 1).

Theorem 4.6. Let $(\Omega, \mu)$ be a $\sigma$-finite measure space. If $f: \Omega \rightarrow X$ is a measurable weakly integrable function. Then $f$ can be represented in the form $f=g+h \mu$-a.e. where $g$ is a bounded Bochner integrable function and $h$ assumes at most a countable number of values in $X$.

\section{ACKNOWLEDGEMENTS}

The authors thank A. Albanese for a suggestion about the end of the proof of Theorem 2.2. The research of the second author was partially supported by the MICINN and FEDER Projects MTM2007-64222 and MTM2010-14909, and by Generalitat Valenciana Project PROMETEO/2008/101.

\section{REFERENCES}

[1] C. Badea and S. Grivaux. Unimodular eigenvalues, uniformly distributed sequences and linear dynamics. Adv. Math. 211 (2007), 766-793.

[2] J. Banasiak. Birth-and-death type systems with parameter and chaotic dynamics of some linear kinetic models. Z. Anal. Anwendungen 24 (2005), 675-690.

[3] J. Banasiak and M. Moszyński. A generalization of Desch-Schappacher-Webb criteria for chaos. Discrete Contin. Dyn. Syst. 12 (2005), 959-972.

[4] F. Bayart and S. Grivaux. Frequently hypercyclic operators. Trans. Amer. Math. Soc. 358 (2006), 5083-5117.

[5] F. Bayart and T. Bermúdez, Semigroups of chaotic operators, Bull. London Math. Soc. 41 (2009), 823-830.

[6] F. Bayart and E. Matheron Dynamics of linear operators. Cambridge Tracts in Mathematics, 179. Cambridge University Press, Cambridge, 2009.

[7] T. Bermúdez, A. Bonilla, J. A. Conejero and A. Peris, Hypercyclic, topologically mixing and chaotic semigroups on Banach spaces, Studia Math. 170 (2005), 57 - 75.

[8] T. Bermúdez, A. Bonilla, and H. Emamirad. Chaotic tensor product semigroups. Semigroup Forum 71 (2005), 252-264.

[9] L. Bernal-González and K.-G. Grosse-Erdmann, Existence and nonexistence of hypercyclic semigroups, Proc. Amer. Math. Soc. 135 (2007), $755-766$. 
[10] A. Bonilla, K.-G. Grosse-Erdmann. Frequently hypercyclic operators and vectors. Ergodic Theory Dynam. Syst. 27 (2007), 383-404.

[11] A. Bonilla, K.-G. Grosse-Erdmann. Frequently hypercyclic operators and vectors ERRATUM. Ergodic Theory Dynam. Syst. 29 (2009), 1993-1994.

[12] J. K. Brooks. Representations of weak and strong integrals in Banach spaces. Proc. Nat. Acad. Sci. U.S.A. 63 (1969), 266-270.

[13] J. A. Conejero, E. M. Mangino. Spectral conditions for hypercyclic $C_{0}$-semigroups. In:J.A.Jaramillo Aguado et al., Function Theory on infinite dimensional spaces - X Conference - Madrid 11-14/12/200\%, Universidad Complutense de Madrid, 2008.

[14] J. A. Conejero, E. M. Mangino, Hypercyclic semigroups generated by Ornstein-Uhlenbeck operators. Mediterr. J. Math. 7 (2010), 101-109.

[15] J. A. Conejero, V. Müller, and A. Peris. Hypercyclic behaviour of operators in a hypercyclic $C_{0}$-semigroup. J. Funct. Anal. 244 (2007), 342-348.

[16] J. A. Conejero and A. Peris, Linear transitivity criteria, Topology Appl. 153 (2005), 767-773.

[17] J. A. Conejero and A. Peris, Hypercyclic translation $C_{0}$-semigroups on complex sectors, Discrete Contin. Dyn. Syst. 25 (2009), 1195-1208.

[18] G. Costakis and A. Peris. Hypercyclic semigroups and somewhere dense orbits. C. R. Math. Acad. Sci. Paris 335 (2002), 895-898.

[19] R. deLaubenfels and H. Emamirad. Chaos for functions of discrete and continuous weighted shift operators. Ergodic Theory Dynam. Systems 21 (2001), 1411-1427.

[20] W. Desch and W. Schappacher, On products of hypercyclic semigroups, Semigroup Forum 7 (2005), 301-311.

[21] W. Desch, W. Schappacher, and G. F. Webb. Hypercyclic and chaotic semigroups of linear operators. Ergodic Theory Dynam. Systems 17 (1997), 793-819.

[22] J. Diestel, J.J. Uhl. Vector measures. Mathematical Surveys, No. 15. American Mathematical Society, Providence, R.I., 1977.

[23] S. El Mourchid. The imaginary point spectrum and hypercyclicity. Semigroup Forum 73 (2006), 313-316.

[24] S. El Mourchid, G. Metafune, A. Rhandi, and J. Voigt. On the chaotic behaviour of size structured cell populations. J. Math. Anal. Appl. 339 (2008), 918-924.

[25] S. Grivaux, A probabilistic version of the frequent hypercyclicity criterion. Studia Math. 176 (2006), 279-290.

[26] S. Grivaux, A new class of frequently hypercyclic operators with applications, Preprint 2010.

[27] K.-G. Grosse-Erdmann and A. Peris. Frequently dense orbits. C. R. Acad. Sci. Paris, Ser. I 341 (2005), 123-128.

[28] L. Ji and A. Weber, Dynamics of the heat semigroup on symmetric spaces, Ergod. Th. Dynam. Sys. 30 (2010), 457-468.

[29] T. Kalmes. On chaotic $C_{0}$-semigroups and infinitely regular hypercyclic vectors. Proc. Amer. Math. Soc. 134 (2006), 2997-3002.

[30] T. Kalmes. Hypercyclic, mixing, and chaotic $C_{0}$-semigroups induced by semiflows. Ergodic Theory Dynam. Systems 27 (2007), 1599-1631.

[31] T. Kalmes. Hypercyclic $C_{0}$-semigroups and evolution families generated by first order differential operators. Proc. Amer. Math. Soc. 137 (2009), 3833-3848.

[32] M. Matsui, M. Yamada, and F. Takeo, Erratum to: "Supercyclic and chaotic translation semigroups" [Proc. Amer. Math. Soc. 131(11): 3535-3546, 2003], Proc. Amer. Math. Soc. 132 (2003), 3751-3752.

[33] G. Metafune. $L^{p}$-spectrum of Ornstein-Uhlenbeck operators. Ann. Scuola Norm. Sup. Pisa Cl. Sci. 30 (2001), 97-124.

[34] R. Rudnicki, Chaos for some infinite-dimensional dynamical systems, Math. Methods Appl. Sci. 27 (2004), 723-738.

[35] C.L. Stewart, R. Tijdeman. On infinite-difference sets. Canad. J. Math. 31 (1979), 897-910.

[36] F. Takeo. Chaos and hypercyclicity for solution semigroups to chaos and hypercyclicity for solution semigroups to some partial differential equations. Nonlinear Anal. 63 (2005), 19431953.

[37] A. Weber, Tensor products of recurrent hypercyclic semigroups, J. Math. Anal. Appl. 351 (2009), 603-606. 
1 Dipartimento di Matematica "Ennio di Giorgi", Università del Salento, I-73100 LECCE, ITALY

E-mail address: elisabetta.mangino@unisalento.it

2 IUMPa, Departament de Matemàtica Aplicada, Edifici 7A, Universitat Politècnica de ValÈncia, E-46022 València, Spain

E-mail address: aperis@mat.upv.es 